\title{
Cluster Abundance Evolution and the Sunyaev-Zeldovich Effect in Various Cosmological Models
}

\author{
Kenji Tomita*) \\ Yukawa Institute for Theoretical Physics, Kyoto University, \\ Kyoto 606-8502, Japan
}

\begin{abstract}
The redshift dependence of observed cluster abundance due to the Sunyaev-Zeldovich Effect (SZE) is studied in various cosmological models including flat and open homogeneous $(\mathrm{CDM})$ models and an inhomogeneous model with a large-scale local void. The PressSchechter formalism is used to derive the abundance at epochs $0<z<2$ and the cluster mass limit $M_{\text {lim }}$ is given from a flux limit for SZE. It is shown that SZE is useful for constraining the cosmological model parameters and the abundance in the inhomogeneous model can be comparable with that in the low-density homogeneous models. The significance of relative difference of abundances in their models is discussed.
\end{abstract}

\section{$\S 1$. Introduction}

The abundance of clusters has been extensively studied by many people in the Xray survey to constrain the cosmological parameters, e.g., Bahcall and Fan, $\frac{1}{1}$ Viana

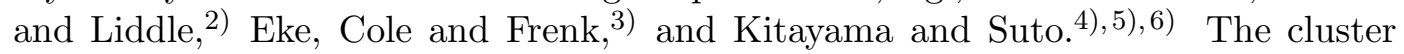
abundance in the submm survey based on the Sunyaev-Zeldovich effect (SZE) has also been studied by Haiman, Mohr and Holder,7] Holder, Haiman and Mohr, [8, ,9] Kitayama, Sasaki and Suto, 10 and Fan and Chiueh.11) This SZE survey is going to be most important to clarify the evolution of clusters and constrain the cosmological parameters.

In this paper we study the cluster abundance in the SZE survey, whose observational condition is set corresponding to the interferometric arrays in the AMIBA project. The cluster mass limit $M_{\text {lim }}$ for deriving the abundance is determined using the expression for the flux $S_{\nu}$ given by Kitayama and Suto, $\stackrel{(5)}{,}$ which is different from that in Fan and Chiueh!11) This brings some difference in the behavior of resulting cluster abundances from that in their paper.

In $\S 2$, we describe the formulation for deriving the cluster abundance in the SZE survey. In $\S 3$ we show the results in various cosmological models. Here we consider first the four representative homogeneous cosmological models: LCDM with $\left(\Omega_{0}, \lambda_{0}\right)=(0.3,0.7)$ and $\Gamma=0.25$, OCDM with $(0.3,0)$ and $\Gamma=0.25$, SCDM with with $(1.0,0)$ and $\Gamma=0.5$, and $\tau \mathrm{CDM}$ with with $(1.0,0)$ and $\Gamma=0.25$, where $\Gamma$ is the CDM shape parameter.

At present most cosmological observations including SDSS, 12 high-redshift supernova $(13),(14), 15),(16)$ and WMAP(17, (18) support a flat homogeneous model with nonzero cosmological constant. However the present observed values of the Hub-

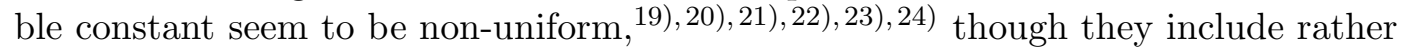

\footnotetext{
*) E-mail address: tomita@yukawa.kyoto-u.ac.jp
} 
large uncertainty, that is, the local median value seems to be larger than that of the global median value by factor $\approx 1.2$. Moreover only one example of supernovae of type Ia with $z>1.5$ has been found ${ }^{26}$ ) and for the data of WMAP 17 , 18) the observed multipole range remains to be $l<900$. Taking this situation into consideration, we take up here also an inhomogeneous model with a large-scale local void as a representative inhomogeneous model, in which there are the inner and outer homogeneous regions I and II and the spherical boundary. $27,, 28), 291,30)$ The motivation of this inhomogeneous model was described in our previous papers. ${ }^{31}$, 32) The cosmological parameters in the two regions are $\left(\Omega_{0}^{\mathrm{I}}, \lambda_{0}^{\mathrm{I}}\right)=(0.3,0), \Gamma^{\mathrm{I}}=0.25, H_{0}^{\mathrm{I}}=70 \mathrm{~km} \mathrm{~s}^{-1}$ $\mathrm{Mpc}^{-1}$ in the inner region (I) and $\left(\Omega_{0}^{\mathrm{II}}, \lambda_{0}^{\mathrm{II}}\right)=(1.0,0), \Gamma^{\mathrm{I}}=0.5, H_{0}^{\mathrm{I}}=H_{0}^{\mathrm{II}} \times 0.82$ in the outer region (II). It is assumed that our observer is at the center for simplicity and the spherical boundary corresponds to the redshift $z_{1}=0.067$. The consistency of spherically symmetric inhomogeneous models with the supernova data was recently discussed and examined by Iguchi et al. ${ }^{33}$ In this section ( $(3)$ we discuss the difference among the abundances in these various models. In section 4 we have concluding remarks.

\section{§2. Number density and SZE}

\subsection{The observed number of clusters}

The comoving number density of clusters of mass $M$ with width $d M$ is

$$
n(M) d M=\left(\frac{2}{\pi}\right) \frac{\rho_{0}}{M} \frac{\delta_{c}(z)}{\sigma_{0}^{2}} \frac{d \sigma_{0}}{d M} \exp \left(-\frac{\delta_{c}^{2}(z)}{2 \sigma_{0}^{2}}\right),
$$

following the Press-Schechter formalism, where $\rho_{0}$ is the present mass density of the universe, $\delta_{c}(z)$ is the linear density threshold for collapse at redshift $z$, and $\sigma_{0}$ is the rms linear density perturbation on the scale corresponding to $M$. The expressions for $\delta_{c}(z)$ in homogeneous models can be seen in Kitayama and Suto's paper ${ }^{[\underline{5})}$ (cf. their Appendix A).

The differential number of clusters is expressed as

$$
\frac{d N}{d z d \Omega}=\frac{d V}{d z d \Omega} \int_{M_{\lim }} n(M) d M
$$

where $d \Omega$ is the solid angle element, $d V$ is the comoving volume element and $M_{\text {lim }}$ is the lower limit of observed cluster mass, which is discussed in the next subsection. Here $d V$ is given by

$$
d V=c t\left[d_{\mathrm{A}}(z)\right]^{2} d \Omega(1+x)^{3}
$$

where $d_{\mathrm{A}}$ is the angular diameter distance. Following Viana and Liddle, ${ }^{2}$ ) we assume $\sigma_{0}$ in the form

$$
\sigma_{0}=\sigma_{8}(z)\left(\frac{R}{8 h^{-1} \mathrm{Mpc}}\right)^{-\gamma(R)}
$$

where $\gamma(R)=(0.3 \Gamma+0.2)\left[2.92+\log _{10}\left(R / 8 h^{-1} \mathrm{Mpc}\right)\right]$ with $M \equiv \frac{4}{3} \pi \rho_{0} R^{3}$, and we have

$$
\sigma_{8}(z)=\sigma_{8}(0) g(\Omega(z), \lambda(z)) /\left[(1+z) g\left(\Omega_{0}, \lambda_{0}\right)\right]
$$


where $g(\Omega, \lambda)$ represents an approximate factor for the growth of linear density perturbations (Carroll, Press and Turner ${ }^{(34)}$ ) given by

$$
g(\Omega, \lambda)=\frac{5}{2} \Omega /\left[\Omega^{4 / 7}-\lambda+(1+\Omega / 2)(1+\lambda / 70)\right]
$$

and $\Omega(z)$ and $\lambda(z)$ are $\Omega$ and $\lambda$ at epochs $z$.

For homogeneous models the observed values of $\sigma_{8}(0)$ have been derived by several groups: for instance, $\sigma_{8}(0) \Omega_{0}^{0.45}=0.53 \pm 0.05$ for $\lambda_{0}=0$ and $\sigma_{8}(0) \Omega_{0}^{0.53}=$ $0.53 \pm 0.05$ for $\Omega_{0}+\lambda_{0}=1$ by Pen ${ }^{(35)}$ and Eke et al. ${ }^{3}$, $\sigma_{8}(0) \Omega_{0}^{0.60}=0.50 \pm 0.04$

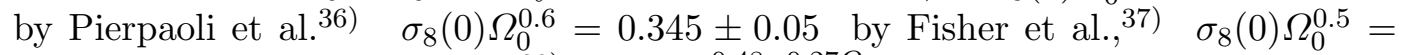
$0.33 \pm 0.03$ by Bahcall et al., ${ }^{38} \sigma_{8}(0) \Omega_{0}^{0.48-0.27 \Omega_{0}}=0.38$ by Viana, Nichol and Liddle ${ }^{39}$ and $\sigma_{8}(0) \Omega_{0}^{0.5}=0.48 \pm 0.12$ by Spergel et al ${ }^{18}$ for the WMAP data. Here we adopt the following two sets of values:

$$
\sigma_{8}(0) \Omega_{0}^{p}=0.4 \text { and } 0.5
$$

where $p$ is 0.45 and 0.53 for models with $\lambda_{0}=0$ and flat models with $\Omega_{0}+\lambda_{0}=1$, respectively.

\subsection{The lower mass limit $M_{\mathrm{lim}}$}

When photons pass through a cluster of hot electrons, the temperature decrement is brought about and the black-body spectrum is distorted due to inverse Compton scattering as

$$
\Delta T / T_{\mathrm{CMB}}=g(x) y,
$$

where the Compton $y$ - parameter is

$$
\begin{gathered}
y \equiv \int n_{e} \sigma_{\mathrm{T}}\left(\frac{k T_{\mathrm{gas}}}{m_{e} c^{2}}\right) d l, \\
g(x) \equiv \frac{x}{\tanh (x / 2)}-4,
\end{gathered}
$$

$x \equiv h \nu /\left(k T_{\mathrm{CMB}}, \nu\right.$ is the CMB photon frequency, $n_{e}$ is the electron number density, $\sigma_{\mathrm{T}}$ is the Thomson cross section, $T_{\text {gas }}$ is the temperature of the cluster gas, and the integration is along the line of sight. If $T_{\text {gas }}>T_{\mathrm{CMB}}$, the flux of CMB photons change from $S_{\nu}^{\mathrm{CMB}}\left(\equiv\left(2 h \nu^{3} / c^{2}\right) /\left(e^{x}-1\right)\right)$ to

$$
S_{\nu}=S_{\nu}^{\mathrm{CMB}} \frac{x e^{x}}{e^{x}-1} g(x) Y,
$$

where

$$
Y=\left[d_{\mathrm{A}}\right]^{-2} \int y d A
$$

and $d A$ is the element of projected area of a cluster. For $\nu=219 \mathrm{GHz}$, we have $g(x)=0$. For $\nu<,>219 \mathrm{GHz}, g(x)<0,>0$, respectively. For the Array for Microwave Background (AMIBA) project (Fan and Chiueh ${ }^{[11}$ ), the parameters are 
$\nu=90 \mathrm{GHz}$ and $x \approx 1.58$, which we take for example in the following. For an isothermal cluster with constant gas mass fraction, we have

$$
Y=\frac{\sigma_{\mathrm{T}}}{2 m_{e} m_{p}}\left[d_{\mathrm{A}}\right]^{-2} f_{\mathrm{ICM}}(1+X) k T_{\mathrm{gas}} M,
$$

where $m_{p}$ is the photon mass, $X$ is the hydrogen mass fraction, and $f_{\mathrm{ICM}} \equiv \Omega_{\mathrm{B}} / \Omega_{0}$

for the present baryon density parameter $\Omega_{\mathrm{B}}$. For the isothermal gas, $T_{\text {gas }}$ is related to the total cluster mass $M$ by

$$
k T_{\text {gas }}=5.2 \gamma(1+z)\left(\frac{\rho_{\mathrm{vir}}(z)}{18 \pi^{2}}\right)^{1 / 3}\left(\frac{M}{10^{15} h^{-1} M_{\odot}}\right) \Omega_{0}^{1 / 3} \mathrm{kev}
$$

as shown by Kitamura, Sasaki and Suto, 10 and the expression of $\rho_{\text {vir }}(z)$ (= the ratio of the mean density of the virialized cluster to the mean density of the universe at each epoch) can be seen in the paper of Kitamura and Sutc (cf. their Appendix A). Here $z$ is the redshift of cluster formation in principle, but it is here regarded as the redshift at the epoch when the cluster is located. Then the total flux of the cluster is

$S_{\nu}=25.5 h(1+z) g(x) \frac{1+X}{2} \times \frac{\Omega_{\mathrm{B}}}{\Omega_{0}^{1 / 3}}\left[\frac{d_{\mathrm{A}}(z)}{c / H_{0}}\right]^{-2}\left(\frac{\rho_{\mathrm{vir}}(z)}{18 \pi^{2}}\right)^{1 / 3}\left(\frac{M}{10^{15} h^{-1} M_{\odot}}\right) \Omega_{0}^{5 / 3} \mathrm{mJy}$,

which is different from the expression in Fan and Chiueh's paper based on Eke, Cole and Frenk ${ }^{\sqrt{3}}$ mainly with respect to the factor $\Omega_{\mathrm{B}} / \Omega_{0}$.

\section{$\S 3 . \quad$ Cluster abundance in various models}

In this section we assume that the limiting flux $\left(S_{\nu}\right)_{\text {lim }}$ is 6.2 mJy corresponding to the AMIBA design, and calculate the limiting mass $M_{\text {lim }}$ using Eq.(2.15). Then by integrating Eq.(2.2), the differential number density of SZE clusters $d N /(d z d \Omega)$ is obtained. In the following we show it in various models described in $\S 1$ : homogeneous models (LCDM, OCDM, SCDM and $\tau \mathrm{CDM}$ ) and an inhomogeneous model with two homogeneous regions I and II, which correspond locally to OCDM and SCDM, respectively. The four homogeneous models correspond to $\left(\Omega_{0}, \lambda_{0}\right)=$ $(0.3,0.7),(0.3,0),(1.0,0)$ and $(1.0,0)$, respectively, and $\Gamma=0.25$ except for SCDM with $\Gamma=0.5$.

\subsection{Homogeneous models}

In Figs. 1 and 2 the behaviors of $d N /(d z d \Omega)$ in four homogeneous models are shown for the two sets $\left(\sigma_{8}(0) \Omega_{0}^{p}=0.5\right.$ and 0.4$)$ given in Eq.(2.7). Here we used $\Omega_{\mathrm{B}} h^{2}=1.70 \times 10^{-2}$. It is found that (1) peaks in the low-density models are at epochs $z=0.17$ and 0.07 for $\sigma_{8}(0) \Omega_{0}^{p}=0.5$ and 0.4 , respectively, and the number density decreases with the increase of $z$ more slowly for larger $\sigma_{8}(0)$, and $(2)$ for $z=0.2$ the number density in LCDM is by factors $>10$ larger than that in SCDM in both cases of $\sigma_{8}(0)$. This trend of latter behaviors is common to that seen in Fig.3

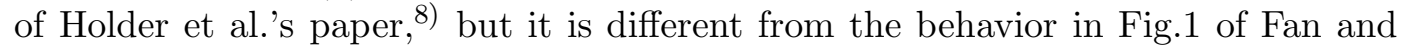


Chiueh.11) This difference comes mainly from whether the factor $\Omega_{\mathrm{B}} / \Omega_{0}$ is included in the expression of $S_{\nu}$ or not.

Next we consider the ratio $r$ of the number $(N(<0.5))$ of clusters with $z<0.5$ to the number $(N(>1.0))$ of clusters with $z>1.0$ in various models, following Fan and Chiueh. In Table 1, the ratios in OCDM and LCDM are shown, while the ratio in SCDM is omitted, because it is too large compared with the other two models. It is found that the ratio $r$ changes sensitively with the value of $\sigma_{8}(0)$ as well as the model parameters. Accordingly the observation of $r$ may give a significant constraint condition on them.

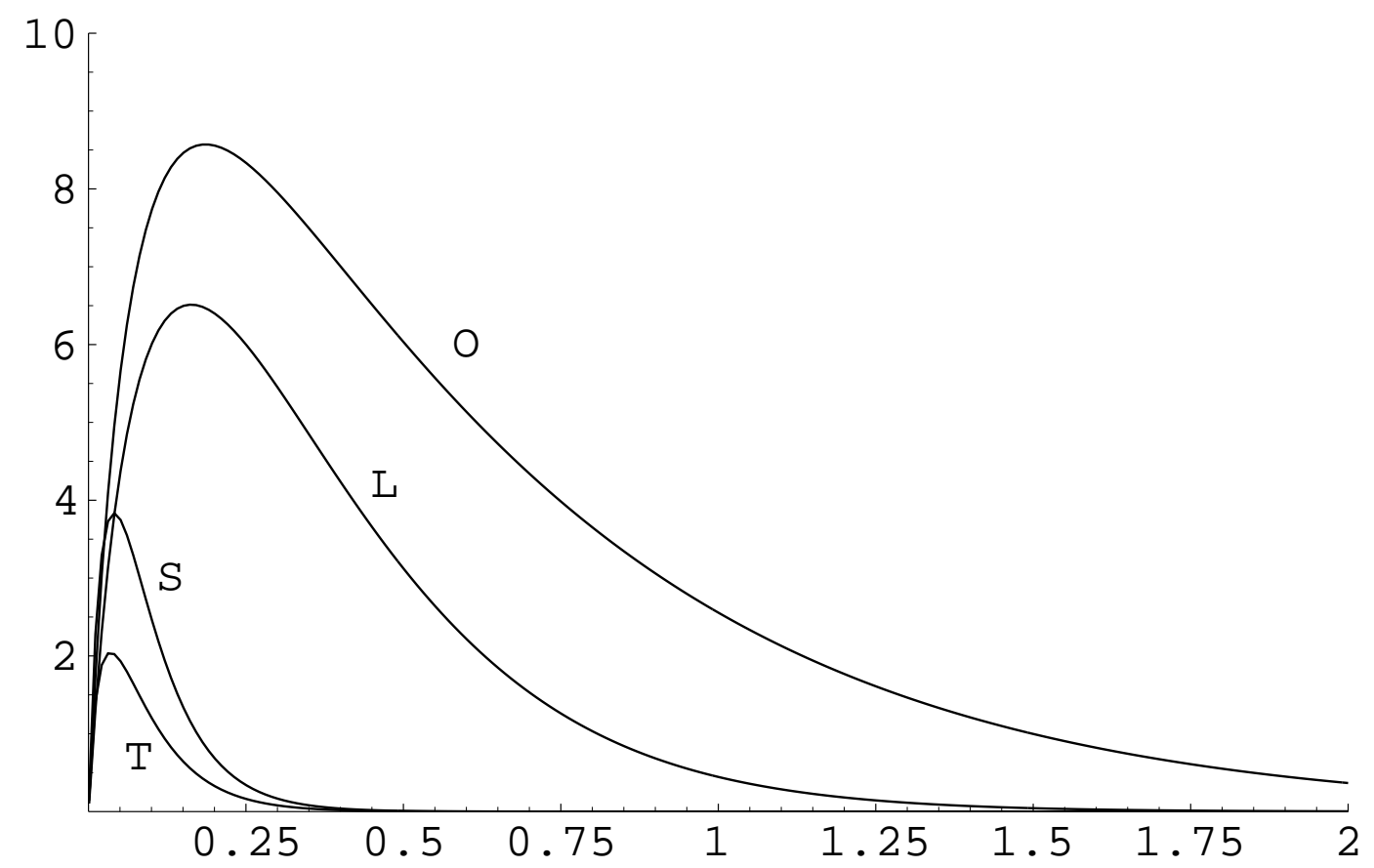

Fig. 1. $d N /(d z d \Omega)$ for $\sigma_{8}(0) \Omega_{0}^{p}=0.5$ in homogeneous models. L and O denote the models (LCDM and OCDM) with $\left(\Omega_{0}, \lambda_{0}\right)=(0.3,0.7)$ and $(0.3,0)$, respectively, in which $\Gamma=0.25$. S and T denote the Einstein-de Sitter models in which $\Gamma=0.5$ and 0.25 , respectively.

\subsection{Inhomogeneous cosmological models}

When we use Eq.(2·15) for $S_{\nu}$, the parameters $\Omega_{0}^{\mathrm{I}}$ and $\Omega_{0}^{\mathrm{II}}$ in homogeneous models are assumed in the inner and outer regions, respectively, as was described in $\S 1$. The baryon density parameters $\Omega_{\mathrm{B}}^{\mathrm{I}}$ and $\Omega_{\mathrm{B}}^{\mathrm{II}}$ have the same ratio $\Omega_{\mathrm{B}}^{\mathrm{II}} / \Omega_{0}^{\mathrm{II}}=$ $\Omega_{\mathrm{B}}^{\mathrm{I}} / \Omega_{0}^{\mathrm{I}}=0.116$, where we used $\Omega_{\mathrm{B}}^{\mathrm{I}} h_{\mathrm{I}}^{2}=0.017$, and $\rho_{\text {vis }}^{i}$ have the values corresponding to $\Omega_{0}^{i}$ for both $i$. For the angular-diameter distance $d_{\mathrm{A}}$, we use the expression in the inhomogeneous model which was given in our previous papers. 27, ,29]

The inner amplitude $\left[\sigma_{8}(0)\right]^{\mathrm{I}}$ is assumed to be given by

$$
\left[\sigma_{8}(0)\right]^{\mathrm{I}}\left(\Omega_{0}^{\mathrm{I}}\right)^{p}=0.5 \text { or } 0.4
$$

so as to be consistent with the local observations of two-point correlation of galaxies, 


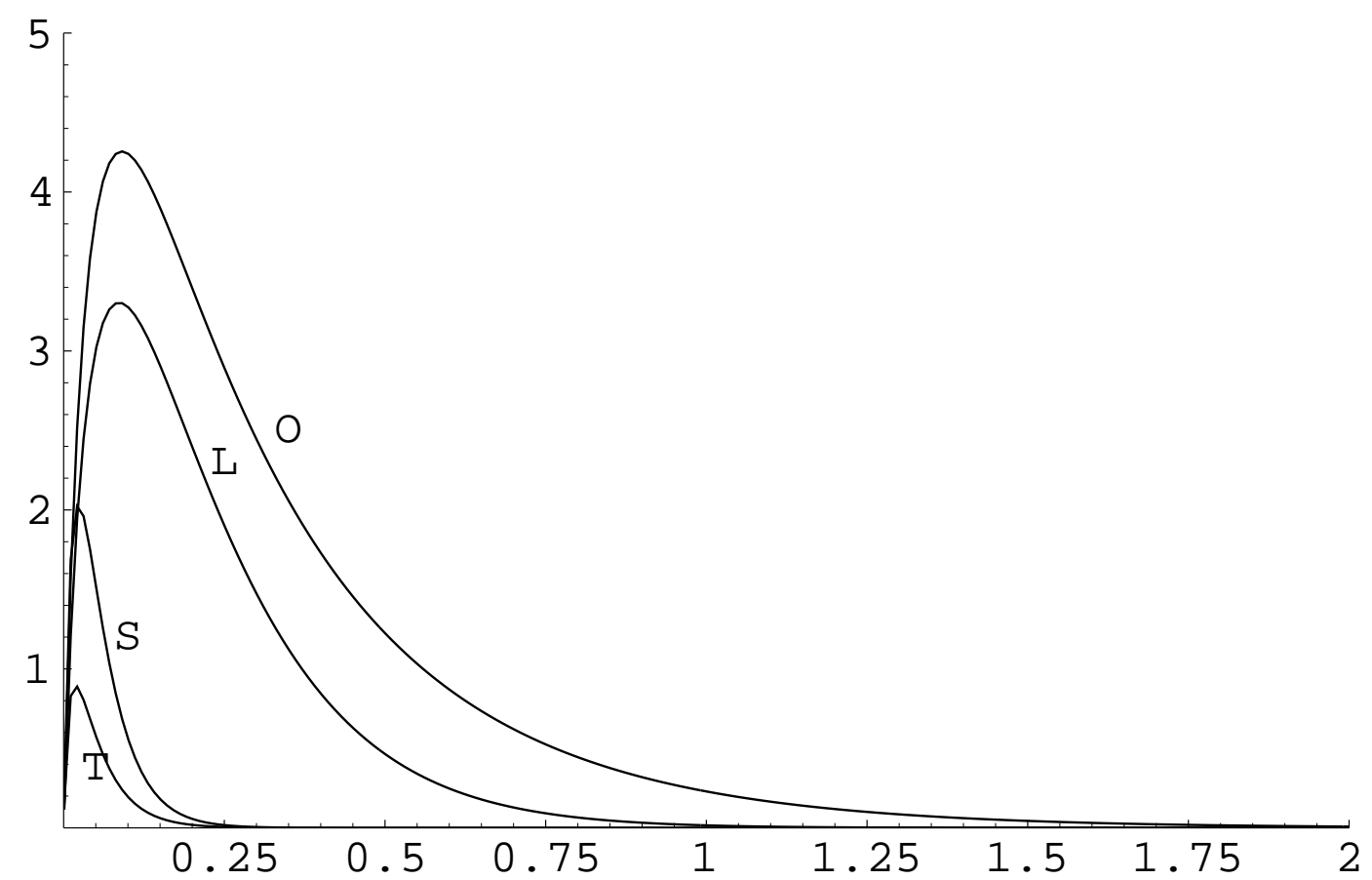

Fig. 2. $d N /(d z d \Omega)$ for $\sigma_{8}(0) \Omega_{0}^{p}=0.4$ in homogeneous models. L and O denote the models (LCDM and OCDM) with $\left(\Omega_{0}, \lambda_{0}\right)=(0.3,0.7)$ and $(0.3,0)$, respectively, in which $\Gamma=0.25$. S and $\mathrm{T}$ denote the Einstein-de Sitter models in which $\Gamma=0.5$ and 0.25 , respectively.

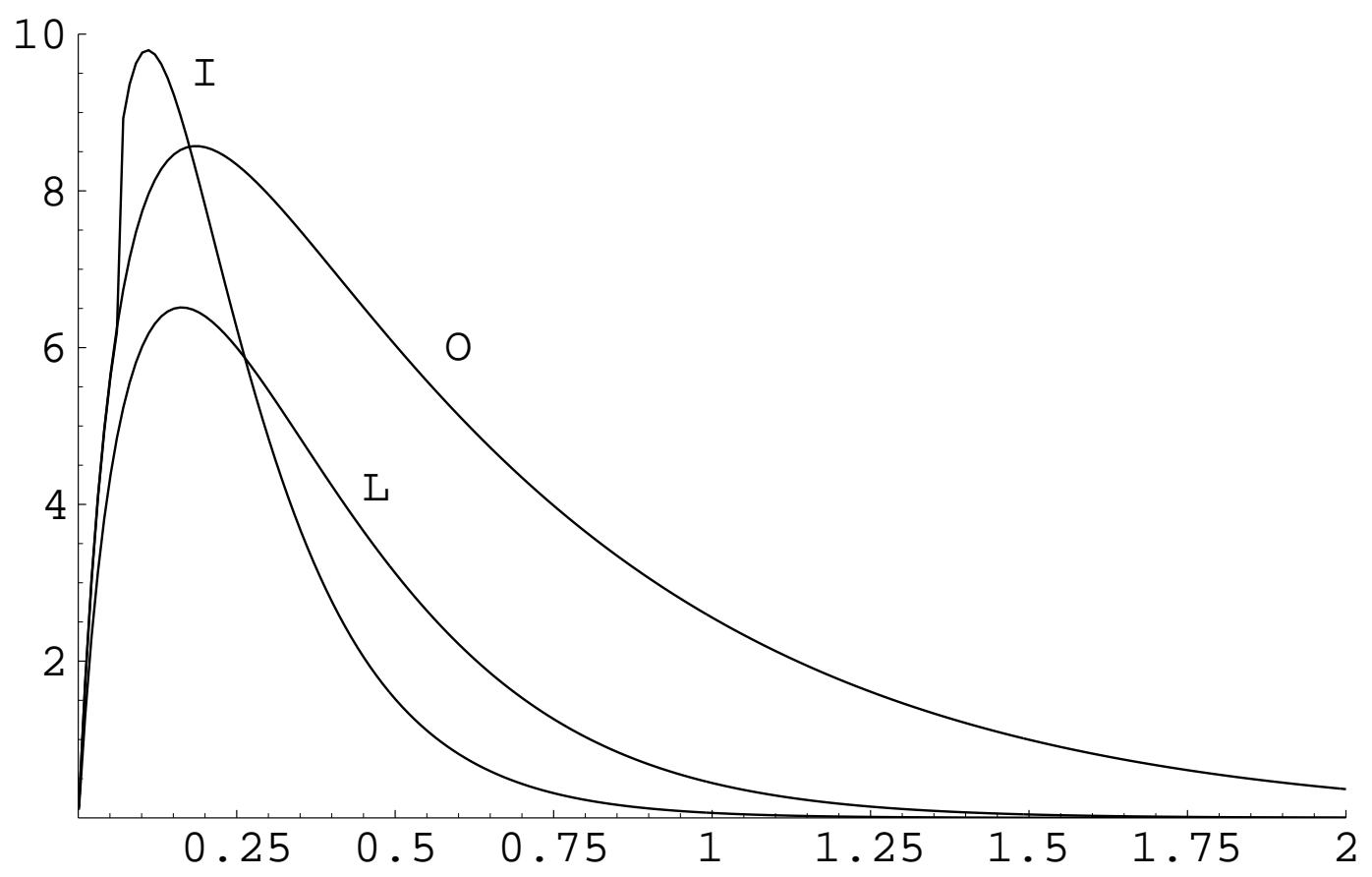

Fig. 3. $d N /(d z d \Omega)$ for $\sigma_{8}(0) \Omega_{0}^{p}=0.5$ in an inhomogeneous model (I) with $\zeta^{2}=2.0$ in comparison with the two homogeneous models $\mathrm{L}$ and $\mathrm{O}$. 


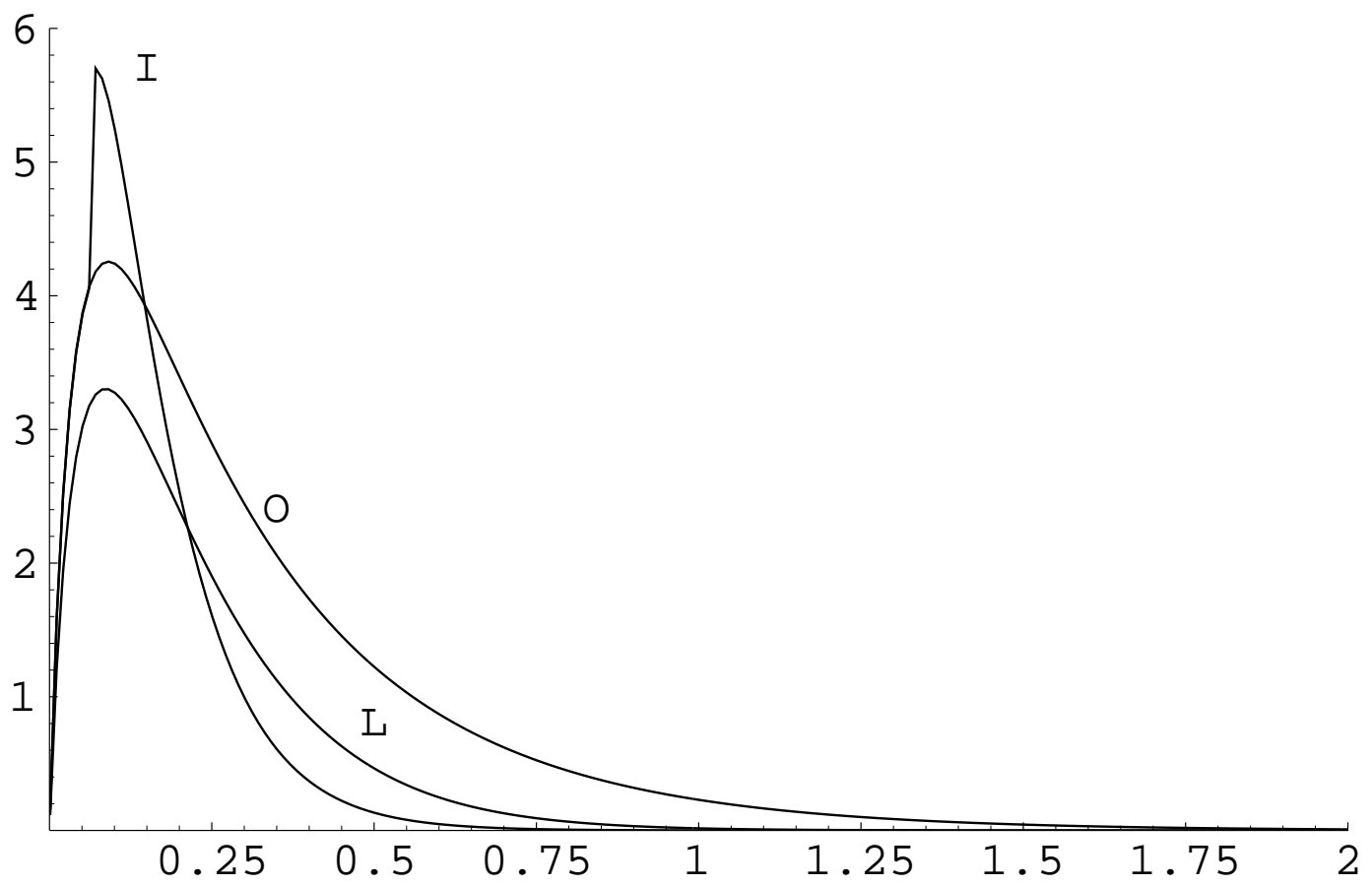

Fig. 4. $d N /(d z d \Omega)$ for $\sigma_{8}(0) \Omega_{0}^{p}=0.4$ in an inhomogeneous model (I) with $\zeta^{2}=2.0$ in comparison with the two homogeneous models $\mathrm{L}$ and $\mathrm{O}$.

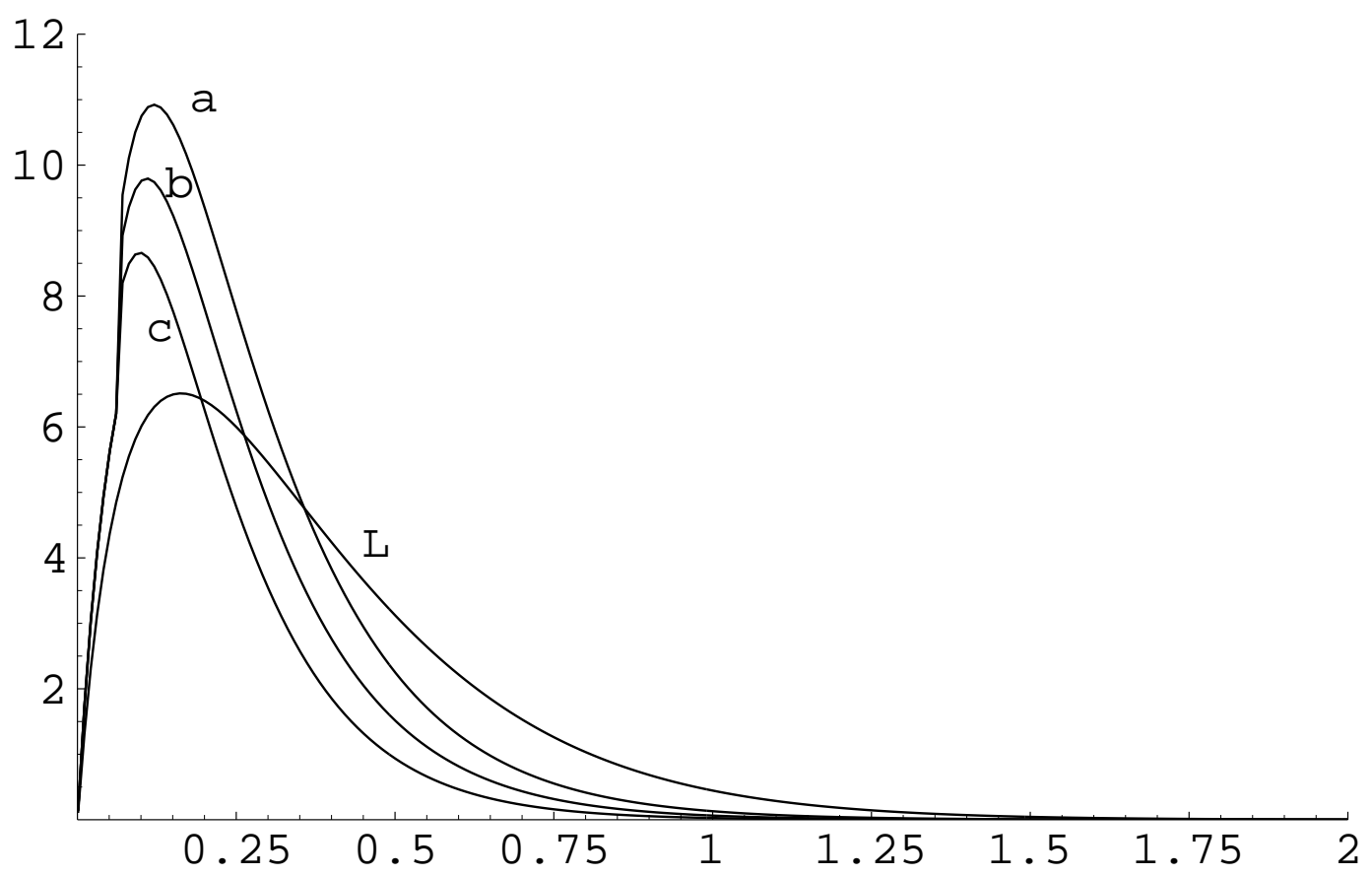

Fig. 5. $d N /(d z d \Omega)$ for $\sigma_{8}(0) \Omega_{0}^{p}=0.5$ in inhomogeneous models with $\zeta^{2}=2.2,2.0$ and 1.8 , which $\mathrm{a}, \mathrm{b}$ and $\mathrm{c}$ denote respectively. 


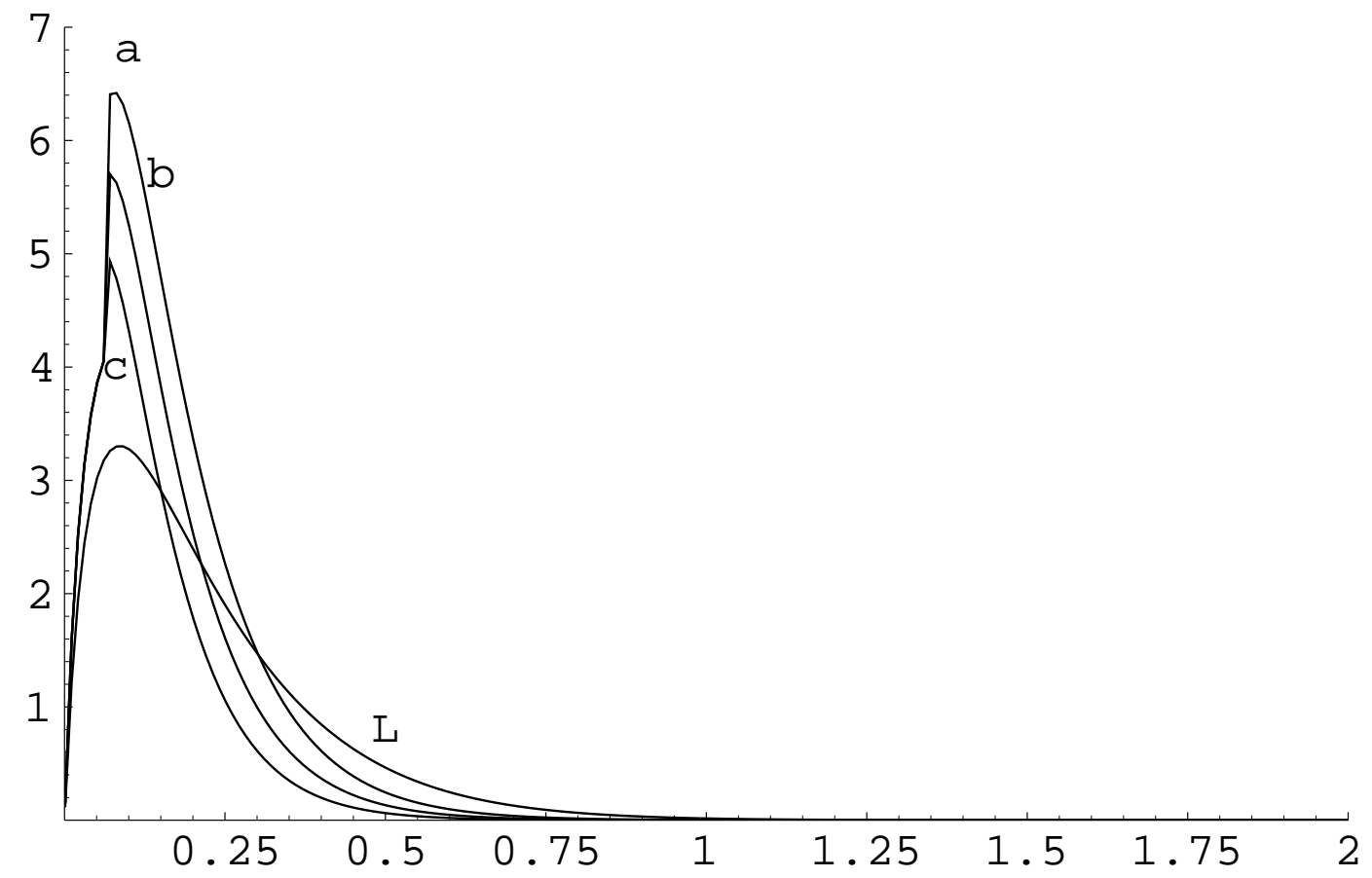

Fig. 6. $d N /(d z d \Omega)$ for $\sigma_{8}(0) \Omega_{0}^{p}=0.4$ in inhomogeneous models with $\zeta^{2}=2.2,2.0$ and 1.8 , which $\mathrm{a}, \mathrm{b}$ and $\mathrm{c}$ denote respectively.

Table I. The ratio $r \equiv N(<0.5) / N(>1.0)$ in various models for the two values of $\sigma_{8}(0)\left(\Omega_{0}\right)^{p}$, where $p$ is 0.45 and 0.53 for models with $\lambda_{0}=0$ and flat models with $\Omega_{0}+\lambda_{0}=1$.

\begin{tabular}{|c|c|c|}
\hline$\sigma_{8}(0)\left(\Omega_{0}\right)^{p}$ & models & ratio $r$ \\
\hline \multirow{5}{*}{0.5} & OCDM $(0.3,0.0)$ & 2.67 \\
& LCDM $(0.3,0.7)$ & 25.0 \\
& Inhom. $\left(\zeta^{2}=2.2\right)$ & 141 \\
& Inhom. $\left(\zeta^{2}=2.0\right)$ & 269 \\
& Inhom. $\left(\zeta^{2}=1.8\right)$ & 586 \\
\hline \multirow{5}{*}{0.4} & OCDM $(0.3,0.0)$ & 19.5 \\
& LCDM $(0.3,0.7)$ & 442 \\
& Inhom. $\left(\zeta^{2}=2.2\right)$ & $4.91 \times 10^{3}$ \\
& Inhom. $\left(\zeta^{2}=2.0\right)$ & $1.33 \times 10^{4}$ \\
& Inhom. $\left(\zeta^{2}=1.8\right)$ & $4.56 \times 10^{4}$ \\
\hline
\end{tabular}

but it is to be noticed with respect to the outer amplitude $\left[\sigma_{8}(0)\right]^{\mathrm{II}}$ that its theoretical amplitudes are by a factor $\sim \sqrt{2}$ larger than $\left[\sigma_{8}(0)\right]_{\text {loc }}$ given by

$$
\left[\sigma_{8}(0)\right]_{\text {loc }}\left(\Omega_{0}^{\mathrm{II}}\right)^{p}=0.5 \text { or } 0.4,
$$

because the theoretical two-point correlation is by a factor $\sim 2$ larger than the observational local values of correlations in the near region, as was shown in a previous paper. ${ }^{32)}$ So we assume that

$$
\left[\sigma_{8}(0)\right]^{\mathrm{II}}=\zeta\left[\sigma_{8}(0)\right]_{\text {loc }}
$$


with $\zeta^{2} \sim 2$. Then $\left[\sigma_{8}(0)\right]^{\mathrm{I}} /\left[\sigma_{8}(0)\right]^{\mathrm{II}} \sim 0.3^{-0.45} / \sqrt{2}=1.22$ for $\zeta^{2}=2$.

In Figs. $3-6$, the behaviors of $d N /(d z d \Omega)$ are shown comparing with those in homogeneous models. In Figs. 3 and 4 (for $\sigma_{8}(0) \Omega_{0}^{p}=0.5$ and 0.4 ) we compared the case $\zeta^{2}=2$ with homogeneous models LCDM and OCDM. It is found from Figs. 1 - 4 that for all $z$ the number density of clusters in the inhomogeneous model is much larger than that in SCDM and that for $z<0.25(0.21)$ for $\sigma_{8}(0) \Omega_{0}^{p}=0.5(0.4)$, respectively, it is larger than that in LCDM, and for $z=0.6$ it is $1 / 2(1 / 3)$ of that in LCDM for $\sigma_{8}(0) \Omega_{0}^{p}=0.5(0.4)$. So it is concluded that SCDM can be ruled out, because of the observed existence of clusters with $z=0.6-0.8$. However the present inhomogeneous model may be consistent with the observed existence of high-redshift clusters, as well as LCDM.@In this inhomogeneous model, it is found moreover that for $z \sim 1$ the cluster number density in the outer is by a factor of $1.5-2.0$ larger than that in the inner region. In Figs. 5 and 6 (for $\sigma_{8}(0) \Omega_{0}^{p}=0.5$ and 0.4 ) the cases with $\zeta=2.2,2.0$ and 1.8 are compared with the case of LCDM. It is found that the cluster number density in the outer region depends highly on the value of $\zeta$, and that it decreases with the increase of $z$ more slowly for larger $\sigma_{8}(0)$. In Table 1 , the ratio $r$ is shown for $\sigma_{8}(0) \Omega_{0}^{p}=0.5$ and 0.4 and for $\zeta^{2}=2.2,2.0$ and 1.8. This shows that the ratio $r$ increases with $\zeta$ and $r$ in the homogeneous model with $\zeta^{2}=2.0$ is by a factor $\sim 10$ larger than $r$ in the LCDM.

\section{$\S 4$. Concluding remarks}

In the inhomogeneous model, the cluster number density in the outer region is larger than that in the inner region, as was shown in the previous section. On the other hand, the galactic number densities in both regions are nearly equal, as was shown by observational galactic surveys and discussed theoretically in inhomogeneous models. Then we find that the number of galaxies within clusters in the outer region is (by a factor of $1.5-2.0$ ) smaller than that in the inner region, and so the mass-luminosity ratio $M / L$ in the outer region may be by the same factor larger than that in the inner region, as long as $L$ of clusters is produced by galaxies within clusters. This result is interesting in connection with the observation of $M / L$ of clusters given by Bahcall and Comerford ${ }^{40}$ (cf. their Table 1), in which we find that the mean value of $M / L$ for clusters with $z>0.07$ is by a factor of $\sim 1.7$ larger than that for clusters with $z<0.067$.

As for the observed values of $\sigma_{8}(0)$, the values from the cluster abundance in the region of $z<0.1^{35}$, (33),, 36 ) seem to be by a factor $\sim 1.5$ larger than the values in the region of $z>0.1 .38$, , 39) The difference of $\sigma_{8}(0)$ in these two regions may be due to the uncertainties, but if it is real, it may suggest the inhomogeneity of $\sigma_{8}(0)$. This is also interesting from the viewpoint of our inhomogeneous models, because $\sigma_{8}(0)$ in the inner region is by a factor $\sim 1.2$ larger than $\sigma_{8}(0)$ in the outer region.

If the observed inhomogeneity of the Hubble constant is true, it cannot be explained in homogeneous models but in inhomogeneous models, while the accelerating behavior of SN1a can be explained also in models with Hubble constant inhomogeneity. The more accurate measurements of the Hubble constant in the gravitational lensing and SZE are desirable! ${ }^{[1]}$ The data of $z=1.7^{(26)}$ is naturally consistent with 
the inhomogeneous model with $\left(\Omega_{0}^{\mathrm{I}}, \Omega_{0}^{\mathrm{II}}\right)=(0.3,1.0), \stackrel{30}{,}$ but the consistency with LCDM was discussed also due to the possible contribution of gravitational lensing to this data. $(42), 43$, ,44) To clarify this difference, we must wait for the day when many data of SN1a with $z>1.5$ are obtained.

\section{Acknowledgments}

The author thanks A. Taruya for helpful discussions on the $\sigma_{8}(0)-\Omega_{0}$ relation. This work was supported by a Grant-in Aid for Scientific Research (No. 12440063) from the Ministry of Education, Science, Sports and Culture, Japan. He also acknowledges use of the YITP computer system for the numerical analyses.

\section{References}

1) N.T. Bahcall and X. Fan, Astrophys. J. 504 (1998), 1.

2) P.T.P. Viana and A.R. Liddle, Mon. Not. R. Astron. Soc. 303 (1999), 535.

3) V.R. Eke, S. Cole and C.S. Frenk, Mon. Not. R. Astron. Soc. 282 (1996), 263.

4) T. Kitayama and Y. Suto, Mon. Not. R. Astron. Soc. 280 (1996), 638.

5) T. Kitayama and Y. Suto, Astrophys. J. 469 (1996), 480.

6) T. Kitayama and Y. Suto, Astrophys. J. 490 (1997), 557.

7) Z. Haiman, J.J. Mohr and G. Holder, Astrophys. J. 553 (2001), 545.

8) G. Holder, et al., Astrophys. J. 544 (2000), 629.

9) G. Holder, Z.Haiman and J.J. Mohr, Astrophys. J. 560 (2001), L111.

10) T. Kitayama, S. Sasaki and Y. Suto, Publ. Astron. Soc. Japan 50 (1998), 1

11) Z. Fan and T. Chiueh, Astrophys. J. 550 (2001), 547.

12) I. Zehavi et al., Astrophys. J. 571 (2002), 172.

13) B. P. Schmidt, N. B. Suntzeff, M. M. Pillips, R. A. Schommer, A. Clocchiatti, R. P. Kirshner, P. Garnavich, P. Challis et al., Astrophys. J. 507 (1998), 46.

14) A. G. Riess, A. V. Filippenko, P. Challis, A. Clocchiatti, A. Diercks, P. M. Garnavich, R. L. Gilliland et al., Astron. J. 116 (1998), 1009.

15) A. G. Riess, A. V. Filippenko, W. Li and B. Schmidt, Astron. J. 118 (2000), 2668.

16) S. Perlmutter, G. Aldering, G. Goldhaber, R. A. Knop, P. Nugent, D. E. Groom, P. G. Castro, S. Deustua et al. , Astrophys. J. 517 (1999), 565.

17) C.L. Bennett et al., astro-ph/0302208

18) D.N. Spergel et al., astro-ph/0302209

19) C.R. Keeton and C.S. Kochanek, Astrophys. J. 487 (1997), 42.

20) F. Courbin et al., Astron. Astrophys. 324 (1997), L1.

21) C.D. Fassnacht, et al., Astrophys. J. 527 (1999), 498.

22) L.L.A. Williams, Astron. J. 119 (2000), 439.

23) M. Tada and T. Futamase, Prog. Theor. Phys. 104 (2001), 971.

24) C.S. Kochanek, Astrophys. J. 578 (2002), 25; Astrophys. J. 583 (2003), 49; astro-ph/0204043

25) E.D. Reese, J.E. Carlstrom, M. Joy, J.J. Mohr, L. Grego and W.L. Holzapfel, Astrophys. J. 581 (2002), 53.

26) A. G. Riess et al., Astrophys. J. 560 (2001), 49.

27) K. Tomita, Astrophys. J. 529 (2000), 26.

28) K. Tomita, Astrophys. J. 529 (2000), 38.

29) K. Tomita, Mon. Not. R. Astron. Soc. 326 (2001), 287.

30) K. Tomita, Prog. Theor. Phys. 106 (2001), 929, astro-ph/0104141

31) K. Tomita, Astrophys. J. 584 (2003), 580.

32) K. Tomita, Prog. Theor. Phys. 108 (2002), 103, astro-ph/0203125

33) H. Iguchi, T. Nakamura and K. Nakao, Prog. Theor. Phys. 108 (2002), 809.

34) S. Carroll, W. Press and E. Turner, Ann. Rev. Astron. Astrophys. 30 (1992), 499.

35) U.-L. Pen, Astrophys. J. 498 (1998), 60.

36) E. Pierpaoli, D. Scott and M. White, Mon. Not. R. Astron. Soc. 325 (2001), 77. 
37) K.B. Fisher et al., Mon. Not. R. Astron. Soc. 266 (1994), 50.

38) N. Bahcall et al., Astrophys. J. 585 (2003), 182.

39) P.T.P Viana, R.C. Nichol and A.R. Liddle, Astrophys. J. 569 (2002), L75.

40) N. Bahcall and J.M. Comerford, Astrophys. J. 565 (2002), L5.

41) M. Oguri and Y. Kawano, Mon. Not. R. Astron. Soc. 338 (2003), L25.

$42)$ G.F. Lewis and R.A. Ibata, Mon. Not. R. Astron. Soc. 324 (2001), L25.

$43)$ E. Mörtsell, C. Gunnarsson and A. Goobar, Astrophys. J. 561 (2001), 106.

44) N. Benítez et al., Astrophys. J. 577 (2002), L1. 